\title{
Chaperone-related immune dysfunction: an emergent property of distorted chaperone networks
}

\author{
Gábor Nardai ${ }^{1}$, Eszter M. Végh ${ }^{1}$, Zoltán Prohászka ${ }^{\mathbf{2}}$ and Péter Csermely ${ }^{\mathbf{1}}$ \\ ${ }^{1}$ Department of Medical Chemistry, Semmelweis University, $\mathrm{H}-1088$ Budapest, Hungary \\ ${ }^{2}$ Department of Internal Medicine III, Semmelweis University, H-1125 Budapest, Hungary
}

\begin{abstract}
Molecular chaperones (heat shock proteins) are important components of cellular networks, such as proteinprotein and gene regulatory networks. Chaperones participate in the folding of immunologically important proteins, presentation of antigens and activation of the immune system. Here, we propose that chaperonerelated immune dysfunction might be more general than was previously thought. Mutations and polymorphism of chaperones and the regulators of their synthesis, heat shock factor-1, chaperone diseases, sick chaperones and chaperone overload might all affect (mostly impairing) immune responses.
\end{abstract}

\section{A central role for chaperones in networks and immune functions}

Chaperones (see Glossary) are ubiquitous, highly conserved proteins, which either sequester damaged proteins, preventing their aggregation, or use a cycle of ATP-driven conformational changes to help the folding of freshly synthesized proteins and the repair of conformational protein damage. Environmental stress (a sudden change in the cellular environment, such as heat shock, to which the cell has not developed a specific adaptive response or does not have time to execute the specific adaptive response) leads to an increase in misfolded proteins and a consequent expression of most chaperones; chaperones are therefore also called heat-shock (Hsps) or stress proteins [1]. Chaperones are important parts of cellular networks, forming complexes with each other, with the numerous co-chaperones regulating their function and with hundreds of other cellular proteins. Examples of these chaperone-related network modules, the recently described 627 members of the yeast $90 \mathrm{kDa} H \mathrm{Hsp}$ (Hsp90) network [2] and the known 160 binding partners of Hsp90 are shown in Figure 1. Behaving as key elements (hubs) in the organization of the protein-interaction networks and genetic-regulatory and membrane or organelle networks of the cell, chaperones promote crosstalk between various signaling pathways, regulate transcriptional networks and might have a role in the coupling of the membrane network of mitochondria, the endoplasmic reticulum and the cell nucleus [3].

Corresponding author: Csermely, P. (csermely@puskin.sote.hu).

Available online 20 December 2005
Chaperones have a prominent role in diverse immune functions. For example, they are associated with the proteasomes; proteasome cap structures themselves have chaperone activity, which enables them to unfold irreversibly damaged proteins and to direct them to the active sites of the proteasomal cavity, where antigenic peptides are generated [4]. Intracellular chaperones in the cytoplasm and in the endoplasmic reticulum have a role in transporting, trimming and presenting antigenic peptides to MHC class I and class II molecules [5]. Extracellular chaperones, released as a result of cell death and taken up by antigen-presenting cells through chaperone receptors 


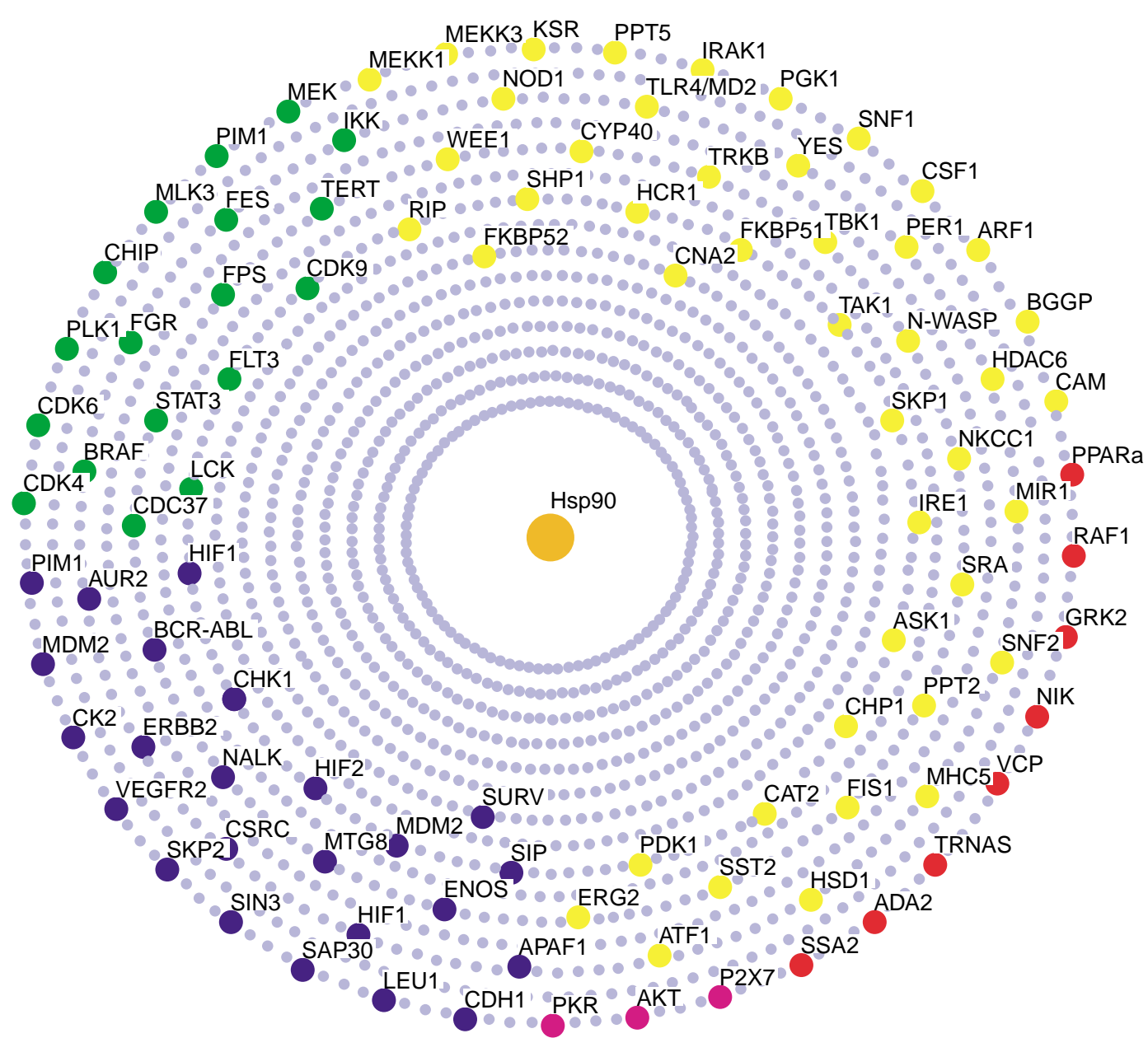

$\overline{T R E N D S ~ i n ~ I m m u n o l o g y ~}$

Figure 1. Involvement of Hsp90-interacting proteins in immune functions. The 627 yeast proteins interacting either physically or genetically with Hsp90 [2] are shown using the Osprey program [50]. Human homologs of yeast network members were included, using the Saccharomyces Genome Database (http://www.yeastgenome.org). A total of 160 known Hsp90 partners were also identified using the Hsp90-interactor table (version 06/2005) downloaded from the Hsp90 Interactors website (http://www.picard.ch/ downloads/Hsp90interactors.pdf), and PubMed (http://www.ncbi.nlm.nih.gov/entrez/query.fcgi) was searched for publications describing immune-related functions of each Hsp90 partner. Possible Hsp90 partners involved in immune regulation (yellow), autoimmune diseases (red) or immune-related malignancies (blue) are highlighted. Green and purple dots represent proteins involved in immune regulation plus malignancies and autoimmune diseases plus malignancies, respectively. Red and purple dots overlap with yellow dots, with the exception of the $60 \mathrm{kDa}$ Ro autoantigen in systemic lupus erythematosus (SSA2), interferon-induced dsRNA-dependent protein kinase (PKR) and the macromolecular His-tRNA-synthetase complex (TRNAS). These overlaps have not been illustrated for the sake of clarity. The 98 immune-related Hsp90 partners are an over-representation because not all interactions are conserved in humans. By contrast, the 98 immune-related Hsp90 partner proteins are an under-representation because many other immune-related Hsp90 partners have multiple names or are excluded from the title, abstract or keyword data on PubMed. Details of Hsp90 partners (including the names of genes, the nature of the interaction, the possible immune-related dysfunction, the available evidence and 144 references) can be found in the online Supplementary Material.

[6], are involved in antigen cross-presentation and the consequent antiviral and anticancer immune response. Although the extent of chaperone-mediated antigenic peptides in cross-presentation has been debated [7,8], the necessity and sufficiency of peptides chaperoned by Hsps for antigen cross-priming of $\mathrm{CD}^{+} \mathrm{T}$ cells was recently shown $[9,10]$. However, the involvement of chaperones in cross-priming might also occur at the proteasomal substrate level [11] or by (chaperoneassisted) autophagy inducing MHC class II presentation of intracellular antigens [12]. Chaperones assist in the quality control of the MHC class I complex, T and B cell receptors and many key proteins of immune signaling [13-15]. The central role of chaperones in cellular networks predicts their links to several other proteins involved in immune functions, such as calcineurin
(CNA2), the cytokine receptor FLT3, the Lck tyrosine kinase, the Toll-like receptor TLR4 and the other 94 examples of binding partners of Hsp90 alone that are highlighted in Figure 1 and in the online Supplementary Material.

Here, we propose that the contribution of chaperones to immune dysfunction is larger than previously thought. Deleterious mutations of chaperones, chaperone polymorphisms, impaired chaperone production, chaperone damage and chaperone overload might all lead to chaperone-related immune dysfunction. This prompts the investigation of immune dysfunction in chaperone deficiencies and folding diseases and suggests novel experiments to assess the role of molecular chaperones in immune function during stress and aging. Because our proposal is related to the function of molecular chaperones 
Table 1. Chaperone mutations associated with immunological dysfunction in humans ${ }^{a, b}$

\begin{tabular}{|c|c|c|}
\hline Mutated chaperone & Primary effect & Immunological alterations \\
\hline Crystallins & Contribution to cataracts and desmin-related myopathy & Unknown \\
\hline $\begin{array}{l}\text { Peptidyl-prolyl cis-trans } \\
\text { isomerase }\end{array}$ & Contribution to Leber's congenital amaurosis & Unknown \\
\hline Hsp60 (mitochondrial) & Contribution to hereditary spastic paraplegia & $\operatorname{lgG} 2$ deficiency [45] \\
\hline Hsp60 (centrosomal) [46] & $\begin{array}{l}\text { Contribution to McKusick-Kaufman and Bardet-Biedl } \\
\text { syndrome }\end{array}$ & Unknown \\
\hline $\begin{array}{l}\text { Co-factor C (a chaperone } \\
\text { for tubulin folding) }\end{array}$ & Contribution to $\mathrm{X}$-linked retinitis pigmentosa & Susceptibility to aberrant cell-mediated immunity [47] \\
\hline $\begin{array}{l}\text { Co-factor E (a chaperone } \\
\text { for tubulin folding) }\end{array}$ & $\begin{array}{l}\text { Contribution to Sanjad-Sakati syndrome, Kenny-Caffey } \\
\text { syndrome and progressive motor neuronopathy }\end{array}$ & $\begin{array}{l}\text { Increased B lymphocytes, decreased } \mathrm{CD}^{+} \text {and } \mathrm{CD}^{+}{ }^{+} \mathrm{T} \\
\text { lymphocytes in Kenny-Caffey syndrome }[48]\end{array}$ \\
\hline Hsp70 (HSPA9b, mortalin) & Contribution to myelodysplastic syndrome [49] & Unknown \\
\hline
\end{tabular}

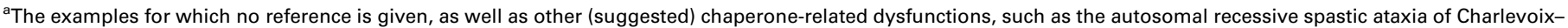
Saguenay or mitochondrial diseases, are from a review by Macario et al. [16].

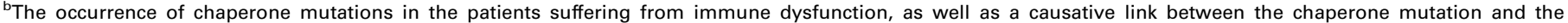
immunological alterations, have not been directly assessed.

as parts of cellular networks, we do not discuss here the extensive and important role of chaperones in immune functions as antigens. It is clear that the various changes in the chaperones we summarize here also affect the immune response against them. Altered immune responses against mutant or damaged chaperones will be subject of exciting studies in the future. Similarly, we do not include the wide range of disorders and pathological states caused by defective chaperones but focus on chaperone-related immune dysfunction. For extensive coverage of chaperone-related pathological states, see [16].

\section{Chaperone polymorphisms and mutations}

Chaperone mutations are primarily exemplified by relatively widespread chaperone single nucleotide polymorphisms, which bring a functional variability to the crossregulated actions of the chaperone network. The $70-\mathrm{kDa}$ Hsp (Hsp70) family shows exceptional polymorphism among human molecular chaperones [17]. The three major Hsp70 genes are located on chromosome 6p21 in the central part of the MHC locus, in close proximity to the genes encoding tumor necrosis factor $\alpha$, lymphotoxin, complement C4 and 21- $\beta$-hydroxylase. These genes regulate immunity, inflammation and the stress response, and are inherited together with MHC class I and class II genes in the form of ancestral haplotypes [18]. An association of Hsp70 polymorphisms and various diseases with inflammatory or autoimmune pathogenesis (such as sepsis, Crohn's disease, Alzheimer's disease and pancreatitis) [19-22], and with acute graft-versus-host disease [23] has been suggested.

Deleterious mutations of various chaperones contribute to the etiology of several diseases as described in Table 1 [14]. These mutations are likely to affect immune function. As an example, IgA deficiency has been shown to occur in the Hsp27-related Charcot-Marie-Tooth disease [24]. We propose that naturally occurring polymorphisms, and mutations of both chaperones and the transcription factor responsible for chaperone induction, heat-shock factor-1 (HSF1), might contribute to immune dysfunction in more cases than those uncovered so far.

\section{Impaired chaperone production}

HSF1 is a member of a transcription factor family and is a key factor in the synthesis of most HSPs in mammals. HSF1 is activated by a sequence of events, starting from the disassembly of the HSF1-chaperone complex in the cytoplasm, through the trimerization, nuclear translocation and phosphorylation of HSF1 and ending with the binding of HSF1 to the respective DNA elements. The final transcriptional events, the dissociation of HSF1 from the DNA and its 'recycling' to the cytoplasm, are also modulated by a large number of cofactors, including molecular chaperones [25]. Recent reports [9,26] showed marked defects in the immune response of HSF1-deficient mice, which are devoid of the transcription factor HSF1 and have an impaired synthesis of most molecular chaperones. One of the reports found that sheep red blood cell-specific IgG production (especially IgG2a production) was approximately half that of the control level following intraperitoneal immunization, and the production of interleukin- 6 and CCL 5 also became impaired in HSF1-deficient mice [26]. Additionally, cross-priming of antigen-specific $\mathrm{CD} 8{ }^{+}$T cells was inefficient when antigen expression was restricted to the nonantigen-presenting cells of HSF1-deficient mice [9].

An example of a more extensive decrease in specific chaperone levels at the cellular level is the deficiency of the endoplasmic reticulum chaperone gp96 (also called Grp94). This caused the inefficient folding of the Toll-like receptors and a subsequent defect in the response of the murine pre-B cell line 70Z/3 [27]. As a further example, the destabilization of the cytoplasmic chaperonin TriC by RNA interference inhibited the peptide loading of MHC class I molecules in the HeLa cell line [28].

In immortalized B cells originally derived from four pairs of identical twins discordant for type 1 diabetes, the decreased expression of the MHC class II invariant chain chaperone led to impaired antigen presentation by the $\mathrm{B}$ lymphocytes of the diabetic patients. The decrease was due to cellular effects of the pathological state and not to specific somatic mutations of the invariant chain chaperone [29]. Chaperone induction is not only specifically, but 
also generally impaired in aging persons [30], which might contribute to age-related immune dysfunction.

At their sites of action, immune cells might often experience a rather pronounced stress - for example, as a result of a local inflammation. Impaired chaperone induction might lead to the dysfunction or apoptosis of cells involved in the immune response [31]. Based on the above examples, we propose that various forms of chaperone deficiency or impaired chaperone induction might make a larger contribution to immune dysfunction in human patients than was previously thought.

\section{Chaperone damage and chaperone overload}

Chaperones have a large hydrophobic surface to recognize damaged proteins, as well as an exceptionally high percentage of naturally disordered regions, which help them to 'catch' their substrates and also to undergo the conformational changes necessary for their assistance in the folding process [32]. These structural features might explain why chaperones are preferentially damaged during aging, by oxidation and other chemical modifications [30], and also show decreased activity during aging [33]. The dysfunction of these chemically damaged, 'sick' chaperones [34] might contribute to the immunodeficient state seen in the elderly. As the extent of this contribution is presently not known, there is a need for studies to analyze the level of chaperone damage in the lymphocytes and dendritic cells of elderly subjects, as well as the use of chemical and overexpressed chaperones to modulate the immune response of these cells.

The levels of damaged or newly folded proteins and the available chaperone capacity are two sides of a carefully balanced system in cells. An excess of chaperone substrates or diminished chaperone content might induce chaperone overload - that is, a relative deficit of available active, unloaded chaperones [35]. Chaperone overload becomes especially large in elderly subjects, in whom protein damage is abundant, and chaperone induction and function are impaired [30]. A special case of chaperone overload occurs in folding diseases, where a misfolded, and usually not degraded, protein sequesters most chaperones. This might contribute to various levels of immune dysfunction in these patients. As an example of this, impaired cytokine production has been reported in Parkinson's disease [36]. However, a causative link between chaperone overload and immune dysfunction has not yet been established, and the misfolded protein might also directly impair various elements of the immune response in Alzheimer's disease [37]. Moreover, in folding diseases, the vast surplus of the misfolded antigen often triggers a specific immune response, which could surpass the putative immune dysfunction. This might occur especially in cases where the misfolded protein is not expressed in cells of the immune system, and therefore might provoke an enhanced specific immune response without a direct inhibition of immune cell functions. Thus, analysis of the contribution of chaperone overload to a possible immune dysfunction will require careful study, preferably in transgenic animals, in which the complex changes of the immune response can be better investigated than in cellular model systems.

\section{Chaperone-related immune dysfunction}

The above scenarios of impaired chaperone function (chaperone polymorphisms, chaperone mutations, impaired chaperone induction, damaged and overloaded chaperones) might all induce an immune dysfunction ranging from the invisible to the pronounced. The hypothetical pathways of chaperone-related immune dysfunction, summarized in Figure 2, are listed below:

(i) Proteasome assembly and proteasome function might become impaired, such that fewer or different antigenic peptides might be generated; proteasomal cross-presentation might also be impaired.

(ii) The transport of antigenic peptides by chaperones might be disturbed in the cytoplasm and into, or within, the endoplasmic reticulum: the spectra of immunogenic peptides might be narrowed to highaffinity binders (which are not necessarily the most immunogenic); fewer or different antigenic peptides might reach the MHC complexes.

(iii) Folding and peptide loading of the MHC complexes might become less efficient or altered; this could have consequences for peripheral tolerance as well as for antiviral and antitumor immunity.

(iv) If these events occur during thymic selection, they might affect positive and negative selection and thus might alter the repertoires of T cells in an organism, thereby modulating the broad antiself-, antiviral- or antitumor reactivity of the host.

(v) Adjuvant ('danger signal') effect of extracellular chaperones, released as a result of cell death, might decrease, leading to inefficient antiviral and antitumor immunity.

(vi) Mutant or damaged chaperones might provoke an altered immune response against themselves.

(vii) $\mathrm{T}$ and $\mathrm{B}$ cell receptors and many other key proteins of the immune system might fold inappropriately; activation and/or anergy of the immune system might become incomplete and slower (Figure $2 b$ ).

In summary, chaperone deficiencies might induce a novel emergent property of cellular networks (a property which cannot be predicted from the behavior of any of the individual network elements): various forms of immune dysfunction.

\section{Possible therapeutic interventions}

Modulation of chaperones or their induction might provide an important therapeutic platform for the development of immunomodulatory drugs. There are several examples of immunosuppressant molecules interacting with molecular chaperones. Cyclophilins and the FK506 immunosuppressant-binding proteins are target chaperones of the widely used immunosuppressants cyclosporin $\mathrm{A}$ and FK506, respectively. The immunosuppressant deoxyspergualine interacts with the structurally similar carboxyterminal regulatory segments of the constitutively expressed form of Hsp70 and with both isoforms of Hsp90. This was proposed as a mechanism to compromise the function of these three chaperones [38]. Similarly, mizoribine, another immunosuppressant, binds to mammalian Hsp60 and inhibits its action [39]. In a similar 
(a)

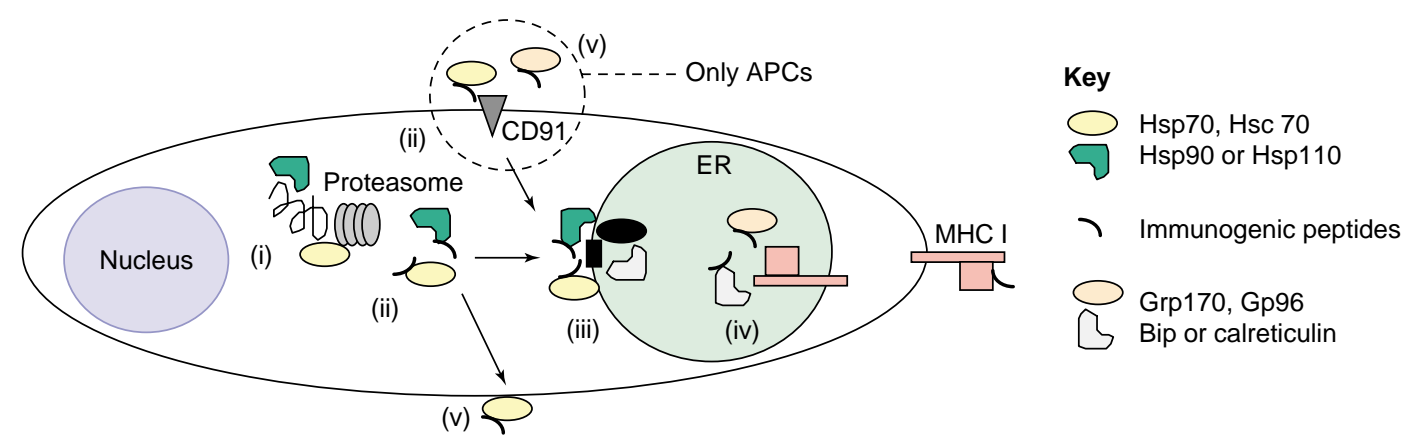

(b)

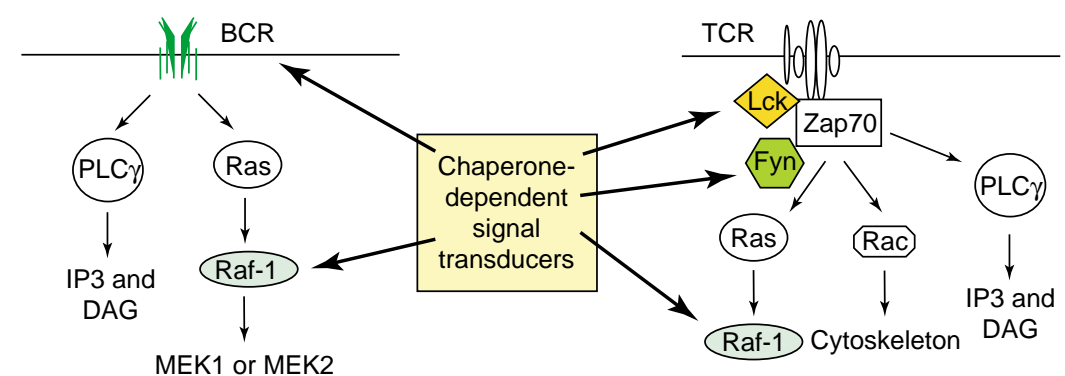

$\overline{T R E N D S ~ i n ~ I m m u n o l o g y ~}$

Figure 2. Hypothetical pathways of chaperone-related immune dysfunctions. (a) (i) Impaired proteasome assembly and function; (ii) antigenic peptide transport through the chaperone relay line is damaged; (iii) untrimmed peptides emerge; (iv) incomplete folding of the MHC I-peptide complex; (v) altered peptide binding and transport by extracellular chaperones; and (vi) altered immune response against chaperones. See text for details. (b) Reduced assistance of molecular chaperones in B and T cell receptor signaling pathways. Molecular chaperones are necessary for the proper folding of B and T cell receptors, as well as of the Raf-1, Lck and Fyn protein kinases that are involved in the signal transduction in B and T cells. Abbreviations: APC, antigen-presenting cell; ER, endoplasmic reticulum; PLC, phospholipase C; IP3, inositol 3-phosphate; DAG diacylglycerol; BCR, B cell receptor; TCR, T cell receptor; MEK, mitogen-activated protein kinase kinase.

context, properly targeted 'bona fide' chaperone inhibitors, such as geldanamycin, might be good candidates for immunosuppressive agents [14].

In the converse situation, where there is a deficiency of chaperone function, overexpression of chaperones or chemical chaperones improves the immune response in animal models [40,41]. Similarly, chaperone inducers and co-inducers [42-44], which are a class of novel therapeutic compounds already being tested in clinical trials, might help to restore compromised immune function in elderly subjects and in other types of chaperone-related immune dysfunction.

\section{Concluding remarks}

Based on the available data in the literature indicating an apparent correlation of various forms of deficient chaperone function with impaired immune response (Table 1), as well as studies on the central role of chaperones in the protein interaction, signaling and transcriptional regulatory networks of the cell, we propose that chaperone mutations, chaperone polymorphisms, impaired chaperone induction, chaperone damage and chaperone overload might all contribute to various levels and forms of immune dysfunction.

Our hypothesis can be tested by a careful analysis of the immune responses of patients suffering from the diseases listed in Table 1, or from folding diseases. If a limited subset of chaperone polymorphisms reveals an extreme dysfunction of the affected chaperones, the immune functions of the respective population should be assessed. Overexpression of damaged proteins, as well as expression of chaperones or HSF1, with dominant-negative mutations in transgenic animals, might all induce an impaired immune response. In all of these pathological or experimental states, a partial reversal of the immune dysfunction might be achieved by the overexpression of chaperones or the addition of chemical chaperones, chaperone inducers or co-inducers. Our hypothesis thus calls for detailed studies of immune functions in chaperone-deficient pathological states, analysis of altered immune responses against mutant or damaged chaperones and the assessment of the contribution of chaperones to immune function in acute, repeated and prolonged stress and aging.

\section{Acknowledgements}

E.M.V. started her research as a member of the Hungarian Research Student Association (http://www.kutdiak.hu). We acknowledge the useful comments of Lajos László and Viktor Müller, George Füst, Pramod K. Srivastava, the referees, as well as the help of Gergely Halász (Semmelweis University) in constructing Figure 1. Work in our laboratory was supported by research grants from the EU (FP6-506850, FP6016003), Hungarian Science Foundation (OTKA-T37357, T46837 and F47281), Hungarian Ministry of Social Welfare (ETT-32/03) and by the Hungarian National Research Initiative (NKFP-1A/056/2004 and KKK0015/3.0).

\section{Supplementary data}

Supplementary data associated with this article can be found at doi:10.1016/j.it.2005.11.009 


\section{References}

1 Young, J.C. et al. (2004) Pathways of chaperone-mediated protein folding in the cytosol. Nat. Rev. Mol. Cell Biol. 5, 781-791

2 Zhao, R. et al. (2005) Navigating the chaperone network: an integrative map of physical and genetic interactions mediated by the hsp90 chaperone. Cell 120, 715-727

3 Sóti, C. et al. (2005) Molecular chaperones as regulatory elements of cellular networks. Curr. Opin. Cell Biol. 17, 210-215

4 Yamano, T. et al. (2002) Two distinct pathways mediated by PA28 and hsp90 in major histocompatibility complex class I antigen processing. J. Exp. Med. 196, 185-196

5 Srivastava, P.K. (2002) Heat shock proteins in innate and adaptive immunity. Nat. Rev. Immunol. 2, 185-194

6 Binder, R.J. et al. (2004) The heat-shock protein receptors: some answers and more questions. Tissue Antigens 64, 442-451

7 Shen, L. and Rock, K.L. (2004) Cellular protein is the source of crosspriming antigen in vivo. Proc. Natl. Acad. Sci. U. S. A. 101, 3035-3040

8 Nicchitta, C.V. et al. (2004) The messenger and the message: gp96 (GRP94)-peptide interactions in cellular immunity. Cell Stress Chaperones 9, 325-331

9 Zheng, H. and Li, Z. (2004) Cutting edge: cross-presentation of cellassociated antigens to MHC class I molecule is regulated by a major transcription factor for heat shock proteins. J. Immunol. 173, 5929-5933

10 Binder, R.J. and Srivastava, P.K. (2005) Peptides chaperoned by heatshock proteins are a necessary and sufficient source of antigen in the cross-priming of CD8+ T cells. Nat. Immunol. 6, 593-599

11 Norbury, C.C. et al. (2004) CD8 + T cell cross-priming via transfer of proteasome substrates. Science 304, 1318-1321

12 Dengjel, J. et al. (2005) Autophagy promotes MHC class II presentation of peptides from intracellular source proteins. Proc. Natl. Acad. Sci. U. S. A. 102, 7922-7927

13 Wright, C.A. et al. (2004) Tapasin and other chaperones: models of the MHC class I loading complex. Biol. Chem. 385, 763-778

14 Schnaider, T. et al. (2000) The Hsp90-specific inhibitor geldanamycin selectively disrupts kinase-mediated signaling events of T-lymphocyte activation. Cell Stress Chaperones 5, 52-61

15 Piatelli, J.M. et al. (2002) Requirement for a hsp90 chaperonedependent MEK1/2-ERK pathway for B cell antigen receptor-induced cyclin D2 expression in mature B lymphocytes. J. Biol. Chem. 277, 12144-12150

16 Macario, A.J.L. et al. (2005) Genetic disorders involving molecularchaperone genes: a perspective. Genet. Med. 7, 3-12

17 Favatier, F. et al. (1997) Variation in hsp gene expression and Hsp polymorphism: do they contribute to differential disease susceptibility and stress tolerance? Cell Stress Chaperones 2, 141-155

18 Miretti, M.M. et al. (2005) A high-resolution linkage-disequilibrium map of the human major histocompatibility complex and first generation of tag single-nucleotide polymorphisms. Am. J. Hum. Genet. 76, 634-646

19 Clarimon, J. et al. (2003) HSP70-2 (HSPA1B) is associated with noncognitive symptoms in late-onset alzheimer's disease. J. Geriatr. Psychiatry Neurol. 16, 146-150

20 Debler, J. et al. (2003) Heat-shock protein HSP70-2 genotypes in patients with Crohn's disease: a more severe clinical course with intestinal complications in presence of the PstI-polymorphism. Eur. J. Med. Res. 8, 120-124

21 Holmes, C.L. et al. (2003) Genetic polymorphism in sepsis and septic shock. Chest 124, 1103-1115

22 Balog, A. et al. (2005) Polymorphism of the TNF-alpha, HSP70-2, and CD14 genes increases susceptibility to severe acute pancreatitis. Pancreas 30, e46-e50

23 Bogunia-Kubik, K. and Lange, A. (2005) HSP70-hom gene polymorphism in allogeneic hematopoietic stem-cell transplant recipients correlates with the development of acute graft-versus-host disease. Transplantation 79, 815-820

24 Williams, L.L. and Penn, G.M. (1979) Selective IgA deficiency in Charcot-Marie-Tooth disease. Am. J. Clin. Pathol. 72, 800-806
25 Morimoto, R.I. (1998) Regulation of the heat shock transcriptional response: cross talk between a family of heat shock factors, molecular chaperones, and negative regulators. Genes Dev. 12, 3788-3796

26 Inouye, S. et al. (2004) Impaired IgG production in mice deficient for heat shock transcription factor 1. J. Biol. Chem. 279, 38701-38709

27 Randow, F. and Seed, B. (2001) Endoplasmic reticulum chaperone gp96 is required for innate immunity but not cell viability. Nat. Cell Biol. 3, 891-896

28 Kunisawa, J. and Shastri, N. (2003) The group II chaperonin TRiC protects proteolytic intermediates from degradation in the MHC class I antigen processing pathway. Mol. Cell 12, 565-576

29 Yan, G. et al. (2003) Impaired processing and presentation by MHC class II proteins in human diabetic cells. J. Immunol. 170, 620-627

30 Sóti, C. and Csermely, P. (2003) Aging and molecular chaperones. Exp. Gerontol. 38, 1037-1040

31 Sreedhar, A.S. and Csermely, P. (2004) Heat shock proteins in the regulation of apoptosis. A comprehensive review. Pharmacol. Ther. 101, 227-257

32 Tompa, P. and Csermely, P. (2004) The role of structural disorder in RNA- and protein chaperone function. FASEB J. 18, 1169-1175

33 Nardai, G. et al. (2002) Chaperone function and chaperone overload in the aged. A preliminary analysis. Exp. Gerontol. 37, 1257-1262

34 Macario, A.J. and Conway de Macario, E. (2005) Sick chaperones, cellular stress and disease. N. Engl. J. Med. 353, 1489-1501

35 Csermely, P. (2001) Chaperone-overload as a possible contributor to 'civilization diseases': atherosclerosis, cancer, diabetes. Trends Genet. 17, 701-704

36 Hasegawa, Y. et al. (2000) Impaired cytokine production by peripheral blood mononuclear cells and monocytes/macrophages in Parkinson's disease. Acta Neurol. Scand. 101, 159-164

37 Fassbender, K. et al. (2004) The LPS receptor (CD14) links innate immunity with Alzheimer's disease. FASEB J. 18, 203-205

38 Nadler, S.G. et al. (1998) Identification of a binding site on Hsc70 for the immunosuppressant 15-deoxyspergualin. Biochem. Biophys. Res. Commun. 253, 176-180

39 Itoh, H. et al. (1999) Mammalian Hsp60 is a major target for the immunosuppressant mizoribine. J. Biol. Chem. 274, 35147-35151

40 Wells, A.D. et al. (1997) Restoration of MHC class I surface expression and endogenous antigen presentation by a molecular chaperone. Scand. J. Immunol. 45, 605-612

41 Ghumman, B. et al. (1998) Chemical chaperones enhance superantigen and conventional antigen presentation by HLA-DM-deficient as well as HLA-DM-sufficient antigen-presenting cells and enhance IgG2a production in vivo. J. Immunol. 161, 3262-3270

42 Yan, D. et al. (2004) Paeoniflorin, a novel heat shock protein-inducing compound. Cell Stress Chaperones 9, 378-389

43 Westerheide, S.D. et al. (2004) Celastrols as inducers of the heat shock response and cytoprotection. J. Biol. Chem. 279, 56053-56060

44 Vigh, L. et al. (1997) Bimoclomol: a nontoxic, hydroxylamine derivative with stress protein-inducing activity and cytoprotective effects. Nat. Med. 3, 1150-1154

45 Woods, G. et al. (1995) Male neonatal death and progressive weakness and immune deficiency in females: an unknown X linked condition. J. Med. Genet. 32, 191-196

46 Galbraith, G.M. et al. (1984) HLA, immunoglobulin allotypes and cellmediated immunity in retinitis pigmentosa: a family study. J. Immunogenet. 11, 165-169

$47 \mathrm{Kim}$, J.C. et al. (2005) MKKS/BBS6, a divergent chaperonin-like protein linked to the obesity disorder Bardet-Biedl syndrome, is a novel centrosomal component required for cytokinesis. J. Cell Sci. 118, $1007-1020$

48 Sabry, M.A. et al. (1998) Kenny-Caffey syndrome is part of the CATCH 22 haploinsufficiency cluster. J. Med. Genet. 35, 31-36

49 Craven, S.E. et al. (2005) Loss of Hspa9b in zebrafish recapitulates the ineffective hematopoiesis of the myelodysplastic syndrome. Blood 105, 3528-3534

50 Breitkreutz, B-J. et al. (2003) Osprey: a network visualization system. Genome Biol. 4, R22 